\title{
Exactitud de la endosonografía rectal en la estadificación tumoral en pacientes con cáncer de recto sin quimio-radioterapia preoperatoria*
}

\author{
Drs. GONZALO URREJOLA S. ${ }^{1}$, JOSÉ GELLONA V. ${ }^{1}$, Int. DAHIANA PULGAR B. ${ }^{2}$, \\ Drs. DANIELLA ESPÍNOLA M. ${ }^{1}$, GONZALO INOSTROZA L. ${ }^{1}$, FELIPE BELLOLIO R. ${ }^{1}$, \\ ALVARO ZÚÑIGA D. ${ }^{1}$, RODRIGO MIGUIELES C. ${ }^{1}$, GEORGE PINEDO M. ${ }^{1 \dagger}$, MARÍA E. MOLINA P. ${ }^{1}$ \\ 1 Unidad de Coloproctología, Departamento de Cirugía Digestiva, Pontificia Universidad Católica de Chile. \\ 2 Interna de Medicina, Facultad de Medicina, Pontificia Universidad Católica de Chile. \\ Santiago, Chile.
}

\begin{abstract}
Accuracy of endoscopic ultrasound in tumor staging of rectal cancer patients not treated with preoperative chemo-radiation

Introduction: Preoperative T staging of rectal cancer is essential for an adequate treatment strategy. Endoscopic ultrasonography (EUS) is one of the available modalities. The reported accuracy of this technique for T staging is variable. This inconsistency might be due to neoadyuvancy, and its downstaging properties. Aim: Determine the accuracy of rectal EUS for T staging of middle and lower rectal tumors in patients not treated with neoadyuvant chemo-radiotherapy. Materials and Methods: Clinical records of all consecutive patients evaluated by rectal EUS between years 2001-2009 in the Catholic University Clinical Hospital were accessed. Of 2.120 patients, 294 had the exam performed for middle or lower rectal cancer. Those who did not receive neoadyuvant chemo-radiation and whose histopathology was available were analyzed. Result: Data was obtained for 69 patients. The overall accuracy of EUS for T staging was 85\%. For T1 tumors, the sensibility, specificity and accuracy were $82 \%, 96 \%$ and $94 \%$ respectively. For T2 tumors the sensibility, specificity and accuracy were $72 \%, 83 \%$ and 78 respectively. For T3 tumors the sensibility, specificity and accuracy were $82 \%, 83 \%$ and $83 \%$ respectively. Conclusion: Rectal EUS continues to be a valuable staging procedure for tumor depth invasion, with an overall accuracy of $85 \%$.
\end{abstract}

Key words: Endoscopic ultrasonography, rectal cancer, accuracy.

\section{Resumen}

Introducción: La estadificación tumoral (T) preoperatoria es esencial para el tratamiento del cáncer de recto. La endosonografía rectal (ER) es una de las modalidades disponibles. La exactitud de esta técnica para la estadificación tumoral es variable en la literatura, y se sospecha que esta inconsistencia se debe a la neoadyuvancia, por el descenso de estadio que esta produce. Objetivo: Analizar la exactitud de la endoso-

*Recibido el 23 de abril de 2012 y aceptado para publicación el 17 de mayo de 2012.

Los autores no refieren conflictos de interés.

Correspondencia: Dra. María Elena Molina P. Marcoleta 340, Santiago, Chile. urregon@gmail.com 
nografía rectal para la estadificación tumoral en pacientes con cáncer de recto medio o inferior que no hayan recibido neoadyuvancia. Material y Método: Se estudió a los pacientes sometidos a endosonografía rectal entre los años 2001-2009 en el Hospital Clínico de la Pontificia Universidad Católica de Chile. De un total de 2.120 pacientes, 294 fueron evaluados por cáncer de recto en tercio medio o inferior. Se analizó el examen de aquellos que no recibieron quimio-radioterapia preoperatoria y se encontraba disponible la anatomía patológica para su comparación. Resultados: Se obtuvo información de 69 pacientes. La exactitud global del examen para la determinación del T fue 85\%. Para la determinación de T1 los valores de sensibilidad, especificidad y exactitud fueron $82 \%$, 96\% y 94\% respectivamente. Para T2 los valores de sensibilidad, especificad y exactitud fueron $72 \%$, 83\% y 78\% respectivamente. Para T3 los valores de sensibilidad, especificidad y exactitud fueron 82\%, 83\% y 83\% respectivamente. Conclusión: La endosonografía rectal sigue siendo un valioso examen para la determinación de la profundidad de invasión tumoral en cáncer de recto con una exactitud global de $85 \%$.

Palabras clave: Endosonografía rectal, cáncer de recto, exactitud.

\section{Introducción}

El cáncer de recto es una patología frecuente en países occidentales, representando el 30\% de los cánceres colorrectales y el 4\% del total de los pacientes con cáncer ${ }^{1}$. En Chile, el año 2006 murieron 368 personas por cáncer rectal². Las complejas relaciones anatómicas del piso pélvico con el recto, así como su mayor tasa de recurrencia y mortalidad comparada con el cáncer de colon, hacen que el manejo del cáncer de recto sea un desafío ${ }^{3-5}$.

El manejo convencional del cáncer de recto en estadios II y III es la quimio-radioterapia neoadyuvante seguida de cirugía resectiva (resección anterior baja o resección abdominoperineal), no obstante la resección total mesorectal como terapia única o la cirugía resectiva seguida de quimio-radioterapia también son opciones válidas. Sin embargo, para tumores con invasión tumoral T1 y T2 seleccionados, el manejo puede ser la resección local seguida de radioterapia adyuvante ${ }^{6}$. Con estas diferencias mayores en el manejo de tumores T2 (estadio I) y T3 (estadio II), la exactitud de la estadificación del $\mathrm{T}$ juega un rol fundamental en la decisión quirúrgica.

Para estadificar al cáncer de recto las herramientas más utilizadas son la tomografía computarizada (TC), la resonancia magnética (RM) y la endosonografía rectal ${ }^{7-9}$. La exactitud de la endosonografía rectal ha variado en la literatura, con excelentes valores iniciales que luego han descendido. Los valores publicados fluctúan entre un 69\% y 97\% para la estadificación tumoral ${ }^{10}$. Esta inconsistencia está en parte determinada por la neoadyuvancia, ya que los estudios publicados que evalúan exactitud, en nuestro conocimiento, incluyen pacientes con y sin neoadyuvancia, y es sabido que esta última produce un descenso del estadio tumoral. Ya que la norma con la que se compara el resultado de la endosonografía es la anatomía patológica de la pieza operatoria, la exactitud del examen se verá afectado si es que la terapia neoadyuvante produjo un descenso del estadio.
El objetivo de nuestro trabajo fue determinar la exactitud de la endosonografía rectal para la estadificación de la invasión tumoral en cáncer de recto medio e inferior, en pacientes sin terapia neoadyuvante.

\section{Material y Método}

En el Departamento de Endoscopia de la Pontificia Universidad Católica de Chile, un total de 2.120 pacientes fueron sometidos a endosonografía rectal entre los años 2001 y 2009. De éstos, 294 fueron evaluados por diagnóstico de cáncer de recto en tercio medio o inferior. Fueron incluidos en el estudio aquellos pacientes que se sometieron a cirugía resectiva del tumor rectal y cuya anatomía patológica estaba disponible para análisis. Se excluyeron todos los pacientes que recibieron quimio-radioterapia preoperatoria. Se estadificó a los tumores según la clasificación TNM (AJCC/UICC 6ta edición, 2002).

Todas las endosonografías rectales fueron realizadas utilizando un ecógrafo B-K Medical, modelo 1850X. El transductor rectal tiene un diámetro de $6 \mathrm{~mm}$. Durante el examen la frecuencia puede ser cambiada de 5,0 a 10,0 MHz, según la penetrancia deseada. La resolución de ambas frecuencias es de $0,9 \mathrm{~mm}$. El transductor se cubre con una vaina de látex, que se llena de suero fisiológico para una mejor transmisión del sonido. Luego de una inspección anal y un examen rectal digital, el transductor es insertado y avanzado bajo control sonográfico (Figuras 1 y 2).

Los resultados de la endosonografía fueron comparados con el estudio histopatológico de la pieza operatoria, que se consideró como estándar de elección. Se calculó la exactitud global de la endosonografía, y para cada estadio de infiltración tumoral por separado se calculó la exactitud, sensibilidad, especificidad y el valor predictivo positivo $\mathrm{y}$ negativo. 


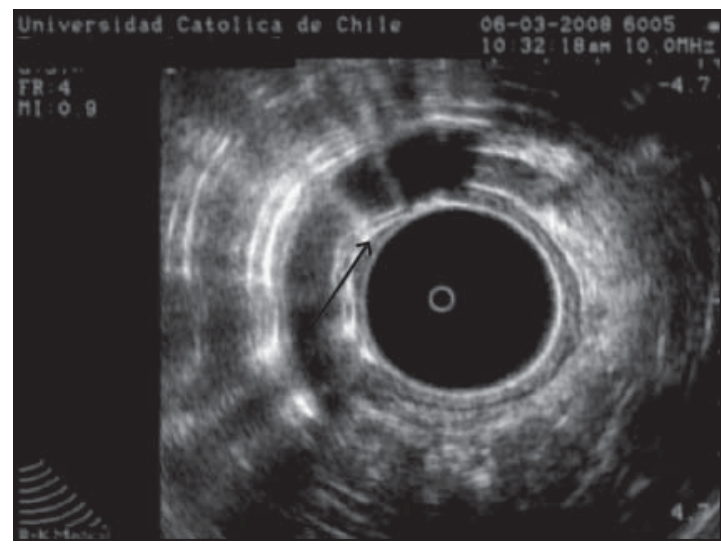

Figura 1. Endosonografía rectal que muestra tumor que invade la submucosa (T1), pero no la muscular propia (Flecha).

\section{Resultados}

De los 294 pacientes evaluados por cáncer rectal en tercio medio o inferior, 69 cumplieron con los criterios de inclusión y no presentaron el criterio de exclusión. Las características basales de los pacientes se exponen en la Tabla 1. La mayoría de los pacientes fueron mujeres (59\%) y se encontraban en estadio I de la enfermedad (45\%). De acuerdo a la histopatología de las piezas operatorias, 11 (16\%) pacientes presentaron tumores T1, 29 (42\%) presentaron tumores T2, 28 (41\%) tumores T3 y un (1\%) paciente presentó un tumor T4. Diecisiete pacientes presentaron compromiso ganglionar (estadio III), pero no recibieron neoadyuvancia ya sea porque tenían tumores parcialmente obstructivos o porque no eran buenos candidatos por el compromiso de su condición general.

La Tabla 2 muestra la comparación entre la estadificación histológica y endosonográfica, donde se aprecia la buena correlación que hubo entre ambas. La exactitud global de la endosonografía rectal para

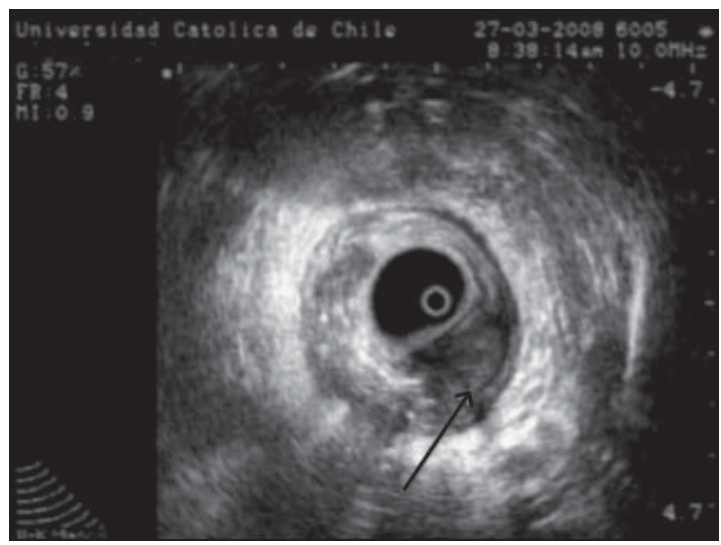

Figura 2. Endonografía rectal que muestra tumor que invade hasta la subserosa, T3 (Flecha).

la estadificación del T fue de 84,9\%. Para tumores T1, la exactitud fue de 94,1\%. Para tumores T2 y T3, la exactitud fue de $78,3 \%$ y $82,6 \%$, respectivamente. La sensibilidad, especificidad y los valores predictivos positivos y negativos para cada estadio T se exponen en la Tabla 3.

\section{Tabla 1. Características basales de los pacientes}

\begin{tabular}{|ll|}
\hline Característica & n \\
\hline n de pacientes & 69 \\
\hline Edad promedio, años (DS) & $68(14,4)$ \\
Hombres & $28(41 \%)$ \\
Estadio TNM & \\
I & $31(45 \%)$ \\
II & $18(26 \%)$ \\
III & $17(25 \%)$ \\
IV & $3(4 \%)$ \\
\hline
\end{tabular}

Tabla 2. Comparación entre estadificación histopatológica y endosonográfica

\begin{tabular}{|c|c|c|c|c|c|c|}
\hline \multirow{6}{*}{ Estadificación endosonográfica } & & \multicolumn{5}{|c|}{ Estadificación histopatológica } \\
\hline & & T1 & T2 & T3 & T4 & Total \\
\hline & $\mathrm{T} 1$ & $9(13 \%)$ & $2(3 \%)$ & 0 & 0 & 11 (16\%) \\
\hline & $\mathrm{T} 2$ & $2(3 \%)$ & $21(30 \%)$ & $5(7 \%)$ & 0 & $28(41 \%)$ \\
\hline & T3 & 0 & $6(9 \%)$ & $23(33 \%)$ & $1(1 \%)$ & $30 \quad(43 \%)$ \\
\hline & Total & 11 (16\%) & $29(42 \%)$ & $28(41 \%)$ & $1(1 \%)$ & 69 (100\%) \\
\hline
\end{tabular}


EXACTITUD DE LA ENDOSONOGRAFÍA RECTAL EN LA ESTADIFICACIÓN TUMORAL EN PACIENTES CON CÁNCER...

Tabla 3. Resultados para sensibilidad (S), especificidad (E), valor predictivo positivo (VPP), valor predictivo negativo (VPN) y exactitud (EX) de la estadificación endosonográfica para cada estadio T

\begin{tabular}{|c|c|c|c|c|c|c|}
\hline \multirow[t]{2}{*}{ Estadio $^{1}$} & \multicolumn{6}{|c|}{ Indicador } \\
\hline & $\mathbf{n}$ & S & $\mathbf{E}$ & VPP & VPN & EX \\
\hline $\mathrm{T} 1$ & 11 & $81,8 \%$ & $96,5 \%$ & $81,8 \%$ & $96,5 \%$ & $94,1 \%$ \\
\hline $\mathrm{T} 2$ & 29 & $72,4 \%$ & $82,5 \%$ & $75 \%$ & $80,5 \%$ & $78,3 \%$ \\
\hline Т3 & 28 & $82,1 \%$ & $82,9 \%$ & $76,7 \%$ & $87,2 \%$ & $82,6 \%$ \\
\hline
\end{tabular}

${ }^{1} \mathrm{~T} 4$ no se incluye al no poder calcular indicadores $\operatorname{con} \mathrm{n}=1$.

\section{Discusión}

Nuestros resultados muestran una buena correlación entre la estadificación endosonográfica e histopatológica para los tumores de recto medio e inferior, con una exactitud global que alcanza un $84,9 \%$.

La ensonografía rectal es una de las técnicas más precisas para la estadificación del cáncer de recto. Algunas publicaciones han mostrado que presenta mejores resultados que la tomografía computarizada para la estadificación del tumor $(\mathrm{T})^{5}$. También presenta ventajas sobre la resonancia magnética al poder diferenciar de manera más certera las distintas capas de la pared rectal. Sin embargo, con los importantes avances tecnológicos de la RM que han mejorado sus resultados, actualmente esta se posiciona como una buena alternativa a la endosonografía para la estadificación tumoral del cáncer de recto ${ }^{11}$. Las principales desventajas de la RM son su alto costo y limitada disponibilidad, además de no presentar tan buenos resultados como la endosonografía cuando se trata de estadificar tumores pequeños ${ }^{12,13}$. Las ventajas que la RM presenta sobre la endosonografía son que no es operador-dependiente, la mejor estadificación de los linfonodos (N) y la capacidad para examinar completamente el mesorecto, hecho relevante ya que al identificar la fascia mesorectal puede determinar el margen de resección circunferencial ${ }^{14}$.

La neoadyuvancia con quimio-radioterapia ha mostrado claros beneficios para los pacientes, cuando se examina del punto de vista de los resultados oncológicos y preservación de la función esfíntérica, al lograr un descenso en el estadio tumoral ${ }^{15,16}$. Sin embargo, es este descenso lo que genera diferencias entre la estadificación preoperatoria y los hallazgos histológicos de la pieza operatoria, afectando la exactitud del examen usado para estadificar al cáncer de recto. Para controlar este efecto confundente en nuestro estudio sólo incluimos pacientes que no recibieron quimio-radioterapia neoadyuvante, y así logramos establecer el verdadero valor de la exactitud, sensibilidad, especificidad y valores predictivos de la endosonografía rectal para la estadificación tumoral preoperatoria del cáncer de recto.

A modo de conclusión, nuestros resultados nos permiten afirmar que la endosonografía rectal presenta una alta exactitud para estadificar el cáncer de recto, con una buena correlación de la invasión tumoral (estadificación del T) con la estadificación histológica. La mejor exactitud (94,1\%) se obtuvo en tumores pequeños (T1), que es donde la endosonografía presenta ventajas comparativas con respecto a otras exploraciones radiológicas, como la TC y la RM.

\section{Referencias}

1. Tjandra JJ, Kilkenny JW, Buie WD, Hyman N, Simmang C, Anthony T, et al. Practice parameters for the Management of Rectal Cancer. Dis Colon Rectum 2005;48:411-23.

2. Instituto Nacional de Estadística de Chile (INE): Estadísticas vitales, informe anual 2006. www.ine.cl/ canales/chile_estadistico/demografia_y_vitales/estadisticas_vitales/pdf/anuarios/vitales_completa_2006.pdf

3. Bedrosian I, Giacco G, Pederson L. Outcome after curative resection for locally recurrent rectal cancer. Dis Colon Rectum 2006;49:175-82.

4. Schiessel R, Novi G, Holzer B. Technique and LongTerm Results of Intersphinteric Resection for Low Rectal Cancer. Dis Colon Rectum 2005;48:1858-67.

5. Harewood GC, Wiersema MJ, Nelson H, Maccarty RL, Olson JE, Clain JE, et al. A prospective, blinded assessment of the impact of preoperative staging on the management of rectal cancer. Gastroenterology 2002;123:24-32.

6. Sengupta S, Tjandra J. Local excision of rectal cancer: what is the evidence? Dis Colon Rectum 2001;44:134561.

7. Guinet C, Buy JN, Ghossain MA, Sezeur A, Mallet A, Bigot JM, et al. Comparison of magnetic resonance imaging and computed tomography in the preoperative staging of rectal cancer. Arch Surg. 1990;125:385-8.

8. Kwok H, Bissett IP, Hill GL. Preoperative staging of rectal cancer. Int J Colorectal Dis. 2000;15:9-20. 
9. Rifkin MD, Ehrlich SM, Marks G. Staging of rectal carcinoma: prospective comparison of endorectal US and CT. Radiology 1989;170:319-22.

10. Thoeni RF. Colorectal cancer: cross sectional imaging for staging of primary tumor and detection of local recurrence. AJR 1991;156:909-15.

11. Nelson H, Petrelli N, Carlin A, Couture J, Fleshman J, Guillem J, et al. Guidelines 2000 for colon and rectal cancer surgery. J Natl Cancer Inst. 2001;93: 583-96.

12. Bartram C, Brown G. Endorectal ultrasound and magnetic resonance imaging in rectal cancer staging. Gastroenterol Clin North Am. 2002;31:827-39.

13. Bipat S, Glas AS, Slors FJ, Zwinderman AH, Bossuyt $\mathrm{PM}$, Stoker J. Rectal cancer, local staging and assessment of lymph node involvement with endoluminal
US, CT, and MR imaging- a meta-analysis. Radiology 2004;232:773-83.

14. Zammit M, Jenkins JT, Urie A, O’Dwyer PJ, Mohillo RG. A technically difficult endorectal ultrasound is more likely to be inaccurate. Colorectal Dis. 2005;7:486-91.

15. Cammà C, Giunta M, Fiorica F, Pagliaro L, Craxì A, Cottone M. Preoperative radiotherapy for resectable rectal cancer: A metaanalysis. JAMA 2000;284:100815.

16. Francois Y, Nemoz CJ, Baulieux J, Vignal J, Grandjean JP, Partensky C, et al. Influence of the interval between preoperative radiation therapy and surgery on downstaging and on the rate of sphincter-sparing surgery for rectal cancer: the Lyon R90-01 randomized trial. J Clin Oncol. 1999;17:2396. 